\title{
Effect of antiviral prophylaxis on influenza outbreaks in aged care facilities in three local health districts in New South Wales, Australia, 2014
}

\author{
Tony Merritt, ${ }^{a}$ Kirsty Hope, ${ }^{b}$ Michelle Butler, ${ }^{a}$ David Durrheim, ${ }^{a}$ Leena Gupta, ${ }^{c}$ Zeina Naijar, ${ }^{c}$ Stephen Conaty, \\ Leng Boonwatt ${ }^{d}$ and Stephanie Fletcher ${ }^{d}$ \\ Correspondence to Tony Merritt (email: Tony.Merritt@hnehealth.nsw.gov.au)
}

Background: There was a record number $(n=111)$ of influenza outbreaks in aged care facilities in New South Wales, Australia during 2014. To determine the impact of antiviral prophylaxis recommendations in practice, influenza outbreak data were compared for facilities in which antiviral prophylaxis and treatment were recommended and for those in which antivirals were recommended for treatment only.

Methods: Routinely collected outbreak data were extracted from the Notifiable Conditions Information Management System for two Local Health Districts where antiviral prophylaxis was routinely recommended and one Local Health District where antivirals were recommended for treatment but not routinely for prophylaxis. Data collected on residents included counts of influenza-like illness, confirmed influenza, hospitalizations and related deaths. Dates of onset, notification, influenza confirmation and antiviral recommendations were also collected for analysis. The Mann-Whitney $U$ test was used to assess the significance of differences between group medians for key parameters.

Results: A total of 41 outbreaks (12 in the prophylaxis group and 29 in the treatment-only group) were included in the analysis. There was no significant difference in overall outbreak duration; outbreak duration after notification; or attack, hospitalization or case fatality rates between the two groups. The prophylaxis group had significantly higher cases with influenza-like illness $(P=0.03)$ and cases recommended antiviral treatment per facility $(P=0.01)$.

Discussion: This study found no significant difference in key outbreak parameters between the two groups. However, further high quality evidence is needed to guide the use of antivirals in responding to influenza outbreaks in aged care facilities.

nfluenza is a notifiable condition in New South Wales (NSW), Australia. Aged care facilities (ACFs) are encouraged to notify influenza outbreaks to their local public health unit ( $\mathrm{PHU})$, where they are recorded in the NSW Notifiable Conditions Information Management System (NCIMS). There were 111 influenza outbreaks notified in NSW ACFs during 2014, the highest on record (Figure 1). ${ }^{1}$ Notified influenza outbreaks require at least one laboratory-confirmed case. The predominant circulating influenza strain in 2014 was $A(H 3 N 2)$. As there was a relatively poor match between the circulating and the seasonal influenza vaccine strain in that year, ${ }^{2}$ an effective antiviral intervention would have been particularly valuable for influenza outbreak control.
There is inconsistent international guidance on the role of antivirals during influenza outbreaks in ACFs, and practice varies both in Australia and internationally. The Communicable Disease Network of Australia guidelines note that "there is a potential role for antiviral medications in the management of influenza outbreaks in residential care facilities as an adjunct to other control measures", 3 and Victorian Health guidelines note that "prophylaxis may be recommended in some cases". ${ }^{4}$ In contrast, antiviral treatment and prophylaxis are routinely recommended for ACF influenza outbreaks in Canada ${ }^{5}$ and the United States of America ${ }^{6}$ based mainly on the findings of observational studies ${ }^{7-11}$ and a randomized controlled trial (RCT) of seasonal prophylaxis. $^{12}$

\footnotetext{
Hunter New England Local Health District, New South Wales, Australia.

Health Protection New South Wales, North Sydney, New South Wales, Australia.

Sydney Local Health District, Camperdown, New South Wales, Australia.

South Western Sydney Local Health District, Liverpool, New South Wales, Australia.

Submitted: 10 August 2015; Published: 19 February 2016

doi: 10.5365/wpsar.2015.6.3.005
} 
Figure 1. Reported aged care facility influenza outbreaks in New South Wales, Australia, 2006 to $2014^{1}$

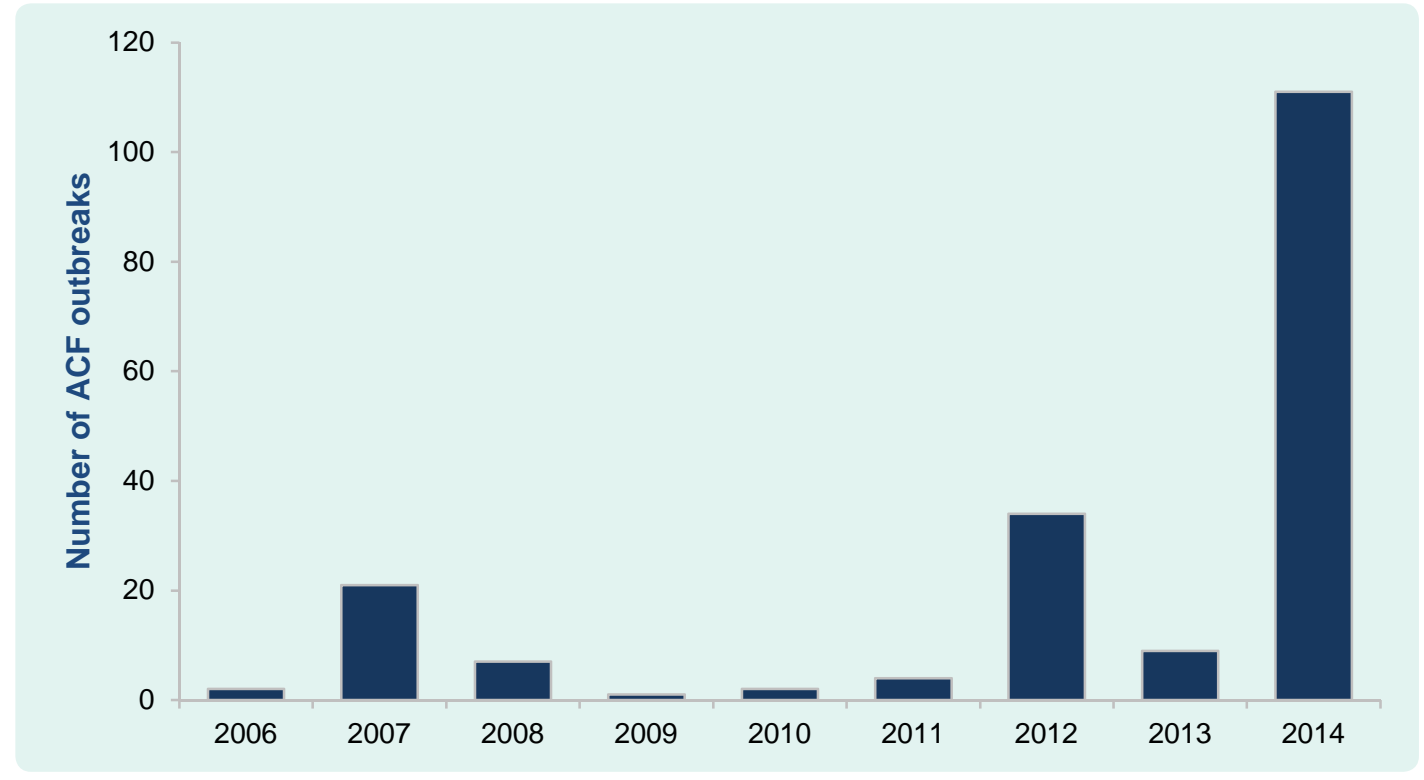

ACF, aged care facility.

The significant resource implications when considering antiviral prophylaxis, including the costs of health staff and medication, further support the need for a strong evidence base for prophylactic antiviral use. To determine the impact of antiviral prophylaxis recommendations in practice, influenza outbreak data were compared for facilities in which antiviral prophylaxis and treatment were recommended and for those in which antivirals were recommended for treatment only.

\section{METHODS}

\section{Study sites}

NSW is divided into 15 Local Health Districts (LHDs), each with a PHU. A convenience sample of two LHDs in which antivirals were routinely recommended to ACFs with influenza outbreaks for both treatment and prophylaxis (prophylaxis group) and one LHD in which antivirals were routinely recommended to ACFs for treatment only (treatment-only group) were included in the analysis.

\section{Outbreak response procedures}

All facilities were provided with routine outbreak management and infection control advice in accordance with Australian guidelines at the time of notification. ${ }^{3}$ In the prophylaxis group, antiviral treatment and prophylaxis were recommended after the first influenza case was confirmed. The PHU sent a letter to the ACF to inform attending medical officers about the outbreak and to recommend oseltamivir treatment for symptomatic cases within 48 hours of onset. Prophylaxis was recommended to other residents for 10 days or until the outbreak was declared over, whichever was the longer period. ${ }^{3}$ If the outbreak was confined to a section or wing that was reasonably separate from the remainder of the facility, a recommendation to offer prophylaxis only to residents in that area was made on some occasions. If ACFs did not have ready access to oseltamivir, a starter pack was provided by the PHU. Prophylaxis was routinely recommended for staff at one LHD.

In the treatment-only group, antiviral treatment of cases, in accordance with national guidelines, ${ }^{3}$ was discussed with ACF staff when the first confirmed case was notified. Routine response measures for both groups included: isolation of ill residents, exclusion of ill staff, cohorting staff to work with either ill or well residents, limiting admission of new residents for the duration of the outbreak, use of appropriate personal protective equipment and enhanced cleaning.

\section{Influenza outbreak data}

All influenza outbreak data for 2014 were extracted from the NCIMS database, including the outbreak details, facility characteristics and key response features (Table 1). All confirmed influenza cases in the 
Table 1. Data extracted from the Notifiable Conditions Information Management System for each influenza outbreak in New South Wales, Australia, 2014

\begin{tabular}{ll}
\hline \multicolumn{1}{c}{ Category } & \multicolumn{1}{c}{ Data } \\
\hline Facility & $\begin{array}{l}\text { Number of residents at risk } \\
\text { Influenza vaccination coverage for residents } \\
\text { Influenza vaccination coverage for staff }\end{array}$ \\
Outbreak & Influenza strain(s) \\
& Number of ILI cases (total cases) \\
& Number of confirmed influenza cases \\
& Onset dates for all ILI cases \\
& Number of cases hospitalized \\
& Number and onset date of related deaths \\
& Date of PHU notification \\
& Date of first positive influenza sample result \\
& Date of PHU visit to the facility \\
& Date of recommendation for use of antiviral prophylaxis \\
& Date of commencement of antiviral prophylaxis \\
& Number of residents recommended antiviral treatment \\
& Number of residents recommended antiviral prophylaxis \\
\hline
\end{tabular}

ILI, influenza-like illness; and PHU, public health unit.

outbreaks were positive by polymerase chain reaction at a laboratory accredited by the National Association of Testing Authorities. ${ }^{13}$ Only illness in residents (but not in health-care workers) was included in the analysis.

Outbreak duration, attack rate, hospitalization rate and case fatality rate were calculated for each facility. Attack rate was further assessed for two time periods: before and after PHU notification. To assess the timeliness of PHU notification, the time from the earliest instance of three influenza-like illness (ILI) cases within a 72 hour period (a potential influenza outbreak) ${ }^{3}$ to $\mathrm{PHU}$ notification was calculated. The time from notification to laboratory confirmation of influenza and the earliest time at which prophylactic antiviral use could be considered was also determined. When confirmation occurred before notification, this period was recorded as zero days. Definitions for the key analysis terms are listed in Table 2.

\section{Statistical methods}

Median and interquartile values were calculated for relevant outbreak parameters and the Mann-Whitney $U$ test was used to assess the significance of differences between group medians. Differences are reported as significant for $P<0.05$. Stata version 11 (StataCorp LP, Texas, USA) was used for all calculations.

\section{RESULTS}

\section{Study population}

The ACFs in the treatment-only and in the prophylaxis groups had comparable numbers of residents at risk (median 85.0 versus 87.5 residents, $P=0.92$ ). Influenza vaccination rates for the two groups did not differ significantly for staff $(50.0 \%$ versus $39.0 \%$, $P=0.11)$ or for residents $(95.6 \%$ versus $98.0 \%$, $P=0.23$ ) (Table 3).

\section{Influenza outbreak profiles}

A total of 41 outbreaks were included in the analysis. The treatment-only group had 29 confirmed influenza outbreaks notified during 2014 , affecting $22.1 \%$ of the 131 ACFs in the district. Antiviral prophylaxis was used for three residents who shared a room with a confirmed case in one large outbreak in this group (with 22 cases and 120 residents at risk). This outbreak was retained as the antiviral prophylactic usage was minimal.

The prophylaxis group had a total of 13 confirmed influenza outbreaks in 2014, affecting $6.2 \%$ of the 210 ACFs in the two districts $(6 / 63,9.5 \%$ and $7 / 147$, $4.8 \%$ in each LHD, respectively). In three outbreaks, prophylaxis was recommended for only part of the ACF 
Table 2. Terms and key analysis parameters used to compare the antiviral prophylaxis and treatment-only groups, New South Wales, Australia, 2014

\begin{tabular}{|c|c|}
\hline Term & Definition \\
\hline $\begin{array}{l}\text { Potential influenza } \\
\text { outbreak }\end{array}$ & Three or more ILI cases in residents within a 72 -hour period. \\
\hline $\begin{array}{l}\text { Confirmed influenza } \\
\text { outbreak }\end{array}$ & $\begin{array}{l}\text { Potential influenza outbreak plus laboratory-confirmed influenza in at least one case. Subsequent to an } \\
\text { influenza outbreak being confirmed, further cases of ILI were considered to be related to the outbreak } \\
\text { whether or not they were laboratory-confirmed. }\end{array}$ \\
\hline Influenza-like illness & $\begin{array}{l}\text { Sudden onset of fever and cough or other respiratory symptoms and one or more systemic symptoms. }{ }^{1} \\
\text { In practice, ILI was loosely defined, and generally a resident was included as an outbreak ILI case if } \\
\text { s/he had acute onset of any respiratory symptom(s) (for example, cough, rhinorrhoea or sore throat). }\end{array}$ \\
\hline $\begin{array}{l}\text { Residents at risk of } \\
\text { infection }\end{array}$ & All residents in the same aged care facility during an influenza outbreak. \\
\hline Outbreak duration & The period from first to last onset date in residents. \\
\hline Linked death & $\begin{array}{l}\text { Death in a resident who was included on an outbreak line list and had a death certificate that included } \\
\text { influenza or respiratory disease as a cause of death or contributing factor. }\end{array}$ \\
\hline Hospitalization rate & Total hospitalized residents/total resident cases (laboratory-confirmed and ILI). \\
\hline Case fatality rate & Linked deaths/total resident cases (laboratory-confirmed and ILI). \\
\hline $\begin{array}{l}\text { Pre-notification attack } \\
\text { rate }\end{array}$ & $\begin{array}{l}\text { Total resident cases (laboratory-confirmed and ILI) up to and including date of PHU notification/total } \\
\text { at-risk residents. }\end{array}$ \\
\hline $\begin{array}{l}\text { Post-notification attack } \\
\text { rate }\end{array}$ & $\begin{array}{l}\text { Total resident cases (laboratory-confirmed and ILI) with onset after date of PHU notification/total at-risk } \\
\text { residents from the day following PHU notification. }\end{array}$ \\
\hline
\end{tabular}

ILI, influenza-like illness; and PHU, public health unit.

resident population. Twelve outbreaks were included in the analysis after excluding one outbreak that was notified too late for prophylaxis.

Influenza was laboratory confirmed in $47.3 \%$ and $41.2 \%$ of the ILI cases in the treatment-only and prophylaxis groups, respectively. The number of confirmed cases per facility was lower in the treatmentonly group than the prophylaxis group (median 5 versus $7, P=0.06)$ as was the number of ILI cases in each outbreak (median 13 versus 23, $P=0.03$ ) (Table 3 ).

Both groups had a similar mix of implicated influenza strains. Influenza A was identified in all outbreaks. The predominant strain was $A / H 3 N 2$. Influenza B was also identified in two outbreaks in the treatment-only group and one in the prophylaxis group (Table 3). Four outbreaks had two different influenza strains identified (two in the treatment-only and two in the prophylaxis group). All outbreaks in the prophylaxis group and $89.7 \%(26 / 29)$ of outbreaks in the treatmentonly group occurred during the influenza season from July to September 2014. Oseltamivir was used for treatment and prophylaxis in all outbreaks.

The outbreak duration (median 9.0 days versus 11.5 days, $P=0.41)$, overall attack rate $(18.3 \%$ versus
$23.9 \%, P=0.15)$, hospitalization rate $(11.1 \%$ versus $14.1 \%, P=0.15)$ and case fatality rate $(0.0$ versus $1.7, P=0.95$ ) were all lower in the treatment-only group than the prophylaxis group, but the differences were not statistically significant. There was also no significant difference between the two groups in preand post-notification attack rates or in outbreak duration after notification $(P>0.05)$ (Table 3 ).

\section{Outbreak responses}

The time from meeting the Communicable Disease Network of Australia's potential influenza outbreak criteria ${ }^{3}$ to PHU notification was similar for the treatmentonly and prophylaxis groups (median 1 day versus 2 days, $P=0.23$ ), as was the time from notification to confirmation (median 1 day versus 1.5 days, $P=0.77$ ). For three outbreaks in the prophylaxis group, influenza confirmation occurred before PHU notification. The median time from antiviral prophylaxis recommendation to medication commencing was 0.5 day (interquartile range, $0.0-1.0$ day) for the prophylaxis group.

Based on the available data, antivirals were used for treatment in a lower proportion of facilities in the treatment-only group (68.8\% versus $83.3 \%$ ), and fewer cases per facility were treated with antivirals in the 
Table 3. Comparison of age care facility influenza outbreak parameters for the antiviral prophylaxis and treatmentonly groups, New South Wales, Australia, 2014

\begin{tabular}{|c|c|c|c|}
\hline & Treatment-only group & Prophylaxis group & $P$-value* \\
\hline ACF outbreaks included & 29 & 12 & NA \\
\hline Antiviral prophylaxis recommended for all residents & 0 & 12 & NA \\
\hline Total residents at risk: ${ }^{\ddagger}$ median (IQR) & $85.0(52.0-123.0)$ & $87.5(66.5-99.5)$ & 0.92 \\
\hline Staff vaccination coverage (\%): ${ }^{\dagger}$ median (IQR) & $50.0(41.5-75.0)$ & $39.0(22.0-50.0)$ & 0.11 \\
\hline Resident vaccination coverage (\%): $:^{\ddagger}$ median (IQR) & $95.6(86.5-98.4)$ & $98.0(95.0-100.0)$ & 0.23 \\
\hline First outbreak onset date & 2 January 2014 & 4 July 2014 & NA \\
\hline Last outbreak onset date & 2 October 2014 & 8 September 2014 & NA \\
\hline Influenza A confirmed (H1, H3, unspecified) & $29(2,18,9)$ & $12(1,7,5)^{\dagger}$ & NA \\
\hline Influenza B confirmed & 2 & 1 & NA \\
\hline ILI outbreak to PHU notification in days: ${ }^{\ddagger}$ median (IQR) & $1.0(0.0-2.0)$ & $2.0(0.5-4.5)$ & 0.23 \\
\hline PHU notification to influenza confirmation in days: ${ }^{\ddagger}$ median (IQR) & $1.0(0.0-3.0)$ & $1.5(0.0-2.5)$ & 0.77 \\
\hline Total confirmed cases: ${ }^{\ddagger}$ median (IQR) & $5(4.0-8.0)$ & $7(6.0-9.0)$ & 0.06 \\
\hline Total ILI cases: ${ }^{\ddagger}$ median (IQR) & $13(9.0-15.0)$ & $23(12.0-28.0)$ & 0.03 \\
\hline Outbreak duration in days: ${ }^{\ddagger}$ median (IQR) & $9.0(7.0-16.0)$ & $11.5(9.5-14.0)$ & 0.41 \\
\hline Total attack rate (\%): ${ }^{\ddagger}$ median (IQR) & $18.3(9.8-25.0)$ & $23.9(17.3-30.6)$ & 0.15 \\
\hline Attack rate (pre-notification) (\%): ${ }^{\ddagger}$ median (IQR) & $9.5(5.7-15.0)$ & $10.9(4.6-20.1)$ & 0.76 \\
\hline Attack rate (post-notification) (\%): ${ }^{\ddagger}$ median (IQR) & $7.8(3.8-13.4)$ & $15.1(7.2-18.2)$ & 0.11 \\
\hline Hospitalization rate (\%): median (IQR) & $11.1(0.0-20.0)$ & $14.1(12.8-25.5)$ & 0.15 \\
\hline Case deaths: ${ }^{\ddagger}$ median (IQR) & $0(0.0-1.0)$ & $1(0-2)$ & 0.21 \\
\hline Case fatality rate (\%): median (IQR) & $0.0(0.0-8.3)$ & $1.7(0.0-7.8)$ & 0.95 \\
\hline Total case deaths & 15 & 12 & NA \\
\hline Duration after PHU notification in days: ${ }^{\ddagger}$ median (IQR) & $6.0(3.0-8.0)$ & $4.5(4.0-8.5)$ & 0.71 \\
\hline Antiviral treatment used in facility & $11 / 16(68.8 \%)$ & $10 / 12(83.3 \%)$ & NA \\
\hline Resident cases recommended antiviral treatment: ${ }^{\ddagger}$ median (range) & $2.5(0.0-12.0)$ & $7.0(0.0-29.0)$ & 0.01 \\
\hline Antiviral prophylaxis used in facility & $1 / 29(3.4 \%)$ & $12 / 12(100 \%)$ & NA \\
\hline Residents recommended antiviral prophylaxis: ${ }^{\ddagger}$ median (IQR) & $0(0.0-3.0)$ & $57.5(7.0-94.0)$ & NA \\
\hline Total residents recommended antiviral prophylaxis & 3 & 544 & NA \\
\hline $\begin{array}{l}\text { Time from antiviral prophylaxis decision to commencement days: } \\
\text { median (IQR) }\end{array}$ & NA & $0.5(0.0-1.0)$ & NA \\
\hline Total deaths 24 hours or more after influenza confirmation & 1 & 3 & NA \\
\hline Facilities with PHU onsite visit & 3 & 3 & NA \\
\hline * Mann-Whitney U test was used. & & & \\
\hline + One outbreak had both influenza A H1 and H3 strains confirmed. & & & \\
\hline$¥ \quad$ Per facility. & & & \\
\hline
\end{tabular}

treatment only group (2.5 versus 7.0 cases, $P=0.01$ ) (Table 3).

\section{DISCUSSION}

We found no significant difference in outbreak attack rate, duration, hospitalization or case fatality rate for those ACFs recommended antiviral treatment alone compared to those recommended antiviral treatment and prophylaxis. The facilities in the two groups had similar numbers of residents and comparable vaccination coverage for both residents and health workers. All outbreaks in the study occurred in NSW during 2014, with the majority in the three-month period between July and September.

The results are consistent with a recent European RCT in an aged care setting ${ }^{14}$ that found no evidence that antiviral prophylaxis during an influenza outbreak reduced the risk of new infections over a four-year period; however, the European study was underpowered. In contrast, an Australian RCT concluded that there was 
"some support for a policy of treatment and prophylaxis with oseltamivir in controlling influenza outbreaks in ACFs", ${ }^{15}$ but the authors in that study also noted that the trial lacked power. Concerns were subsequently raised that one of the three control outbreaks in that study, in which there was a delayed and incomplete intervention, should be excluded. With that outbreak removed, the apparent beneficial effect of antiviral prophylaxis disappeared. ${ }^{16} \mathrm{~A}$ subsequent review by the Academy of Medical Sciences in the United Kingdom in 2015 concluded that there was inadequate evidence to "inform a single approach for prophylaxis in care homes" and that "further research is needed to inform decisions on whether or not to use [antivirals] in prophylaxis in care homes". 17

Some important potential confounding factors were considered in our analysis, including differences in the timeliness of notification, outbreak severity and the thoroughness of interventions between the two groups. No significant difference in the timeliness of PHU notification between the two groups was identified. Outbreak severity was assessed in several ways in this study. The attack rate for the period up to notification did not differ significantly between groups, which is consistent with the two groups having similar overall severity. However, there were some other indications that outbreaks may have been more severe in the prophylaxis group, with median values for overall attack rate and hospitalization rates higher than in the treatment-only group, although neither was significantly different.

A higher proportion of facilities in the treatmentonly LHD reported having influenza outbreaks in 2014. There may have been more outbreaks in this district or there may have been relative underreporting of outbreaks in the prophylaxis districts. An audit of laboratory notifications for influenza in one LHD in the prophylaxis group identified seven ACFs with three or more linked cases of confirmed influenza and a further 15 facilities with one or two cases of confirmed influenza that did not report an outbreak to the PHU in 2014 (South Western Sydney LHD, unpublished data, 2014). Data were not available to assess whether non-reported outbreaks were less severe than those that were notified.

There was also limited capacity to assess the thoroughness of interventions. The same national response guidelines ${ }^{3}$ were used for general infection control advice, but it was not possible to further explore the comparability and completeness of interventions at ACFs. Detailed data on non-antiviral outbreak measures and the extent to which antiviral recommendations were implemented by ACFs were not available. Additional information on antiviral use in future influenza seasons would enhance the analysis of routinely collected ACF outbreak data.

We found four outbreaks had two different influenza strains, indicating multiple importations into those facilities. Other outbreaks may have had unidentified multiple importations with potential impact on the course of the outbreak. Influenza was confirmed in less than half of the ILI cases in the treatment-only and prophylaxis groups. Some residents with ILI may have been infected with other pathogens that affected the analysis.

This was an observational study and is subject to several limitations. The use of antiviral prophylaxis was neither randomized nor blinded, and systematic differences between groups could have confounded the analysis. Notification timeliness, outbreak severity and thoroughness of intervention have been considered in detail, and the overall outbreak profile was similar for the two groups. However some parameters were not considered, including staff illness data, the outbreak setting (some occurred in high dependency units or semi-independent hostel settings), residents' demographics and co-morbidities and the overall resident acuity.

\section{CONCLUSIONS}

Our analysis did not find evidence that a policy of recommending prophylactic antivirals in ACF influenza outbreaks reduced attack rate, outbreak duration, hospitalization rate or case fatality rate during the 2014 influenza season in NSW. Despite the study limitations, the absence of any differences between groups suggests that any effect of antiviral prophylaxis in practice is likely to have been small or negligible. There is a need for further high quality evidence to guide use of antivirals in influenza outbreak response in ACFs.

\section{Conflict of interest}

None declared. 


\section{Funding}

None.

\section{Acknowledgments}

The authors would like to acknowledge the many public health staff from the Hunter New England, Sydney and South Western Sydney Public Health Units and all of the aged care facility and laboratory staff who were involved in the identification and management of these outbreaks.

\section{References}

1. Influenza Monthly Epidemiology Report NSW. New South Wales, NSW Health Department, 2015 (http://www.health.nsw.gov. au/Infectious/Influenza/Documents/2015/January-flu-report.pdf, accessed 8 January 2016).

2. Vyas A et al. Influenza outbreak preparedness, vaccine effectiveness and antigenic drift: lessons from outbreaks in residential care facilities in 2014. Communicable Diseases Intelligence, 2015, 39:E204-207.

3. Communicable Diseases Network Australia. A Practical Guide to assist in the Prevention and Management of Influenza Outbreaks in Residential Care Facilities in Australia. Canberra, The Department of Health, 2009 (http://www.health.gov.au/internet/ main/publishing.nsf/Content/cdna-flu-guidelines.htm, accessed 8 January 2016).

4. Respiratory illness in residential and aged care facilities: guidelines and information kit. Canberra, Department of Health and Human Services, 2014 (https://www2.health.vic. gov.au/about/publications/researchandreports/Respiratory\%20 illness $\% 20$ in $\% 2$ residential\%20and $\% 20$ aged $\% 20$ care $\% 20$ facilities\%20-\%20Guidelines\%20and\%20information\%20kit, accessed 8 January 2016).

5. Aoki FY et al. The use of antiviral drugs for influenza: Guidance for practitioners 2012/2013. The Canadian Journal of Infectious Diseases \& Medical Microbiology = Journal Canadien des Maladies Infectieuses et de la Microbiologie Medicale / AMMI Canada, 2012, 23:e79-92. pmid:24294283

6. Interim Guidance for Influenza Outbreak Management in Long-Term Care Facilities. Atlanta, Centers for Disease Control and Prevention, 2011 (http://www.cdc.gov/flu/ professionals/infectioncontrol/ltc-facility-guidance.htm, accessed 8 January 2016).
7. Hota S, McGeer A. Antivirals and the control of influenza outbreaks. Clinical Infectious Diseases, 2007, 45:1362-1368. doi:10.1086/522661 pmid:17968836

8. Bowles SK et al.; Oseltamivir Compassionate Use Program Group. Use of oseltamivir during influenza outbreaks in Ontario nursing homes, 1999-2000. Journal of the American Geriatrics Society, 2002, 50:608-616. doi:10.1046/j.1532-5415.2002.50153.x pmid:11982659

9. Monto AS et al. Detection and control of influenza outbreaks in well-vaccinated nursing home populations. Clinical Infectious Diseases, 2004, 39:459-464. doi:10.1086/422646 pmid:15356805

10. Harper SA et al; Expert Panel of the Infectious Diseases Society of America. Seasonal influenza in adults and children-diagnosis, treatment, chemoprophylaxis, and institutional outbreak management: clinical practice guidelines of the Infectious Diseases Society of America. Clinical Infectious Diseases, 2009, 48:1003-1032. doi:10.1086/598513 pmid:19281331

11. Fiore $A E$ et al; Centers for Disease Control and Prevention (CDC). Antiviral agents for the treatment and chemoprophylaxis of influenza - recommendations of the Advisory Committee on Immunization Practices (ACIP). Morbidity \& Mortality Weekly Report Recommendations \& Reports, 2011, 60:1-24. pmid:21248682

12. Peters PH Jr et al. Long-term use of oseltamivir for the prophylaxis of influenza in a vaccinated frail older population. Journal of the American Geriatrics Society, 2001, 49:1025-1031. doi:10.1046/ j.1532-5415.2001.49204.x pmid:11555062

13. National Association of Testing Authorities. Australian laboratory accreditation standards. Sydney, National Association of Testing Authorities, Australia, 2015 (http://www.nata.com.au/nata/, accessed 8 January 2016).

14. van der Sande MA et al. Effectiveness of post-exposition prophylaxis with oseltamivir in nursing homes: a randomised controlled trial over four seasons. Emerging Themes in Epidemiology, 2014 11:13. doi:10.1186/1742-7622-11-13 pmid:25210532

15. Booy $R$ et al. Treating and preventing influenza in aged care facilities: a cluster randomised controlled trial. PLoS ONE, 2012, 7:e46509. doi:10.1371/journal.pone.0046509 pmid:23082123

16. Krumholz HM, Jackevicius CA, Ross JS. Tamiflu: 14 flu seasons and still questions. BMJ (Clinical Research Edition), 2013, 346:f547. pmid:23355545

17. Use of neuraminidase inhibitors in influenza. London, The Academy of Medical Sciences and Wellcome Trust, 2015 (http:// www.wellcome.ac.uk/stellent/groups/corporatesite/@policy communications/documents/web_document/wtp059874.pdf, accessed 8 January 2016). 